\title{
COOPERATIVAS AGROALIMENTARIAS Y EXPORTACIÓN. EL PROCESO DE INTERNACIONALIZACIÓN DE LA COOPERATIVA DEL VALLE DE LOS PEDROCHES (COVAP) ${ }^{1}$.
}

POR

\author{
Fernando J. FUENTES GARCÍA ${ }^{2}$ \\ Sandra $\mathrm{M}^{\mathrm{a}} \mathrm{SÁNCHEZ} \mathrm{CAÑIZARES}{ }^{3} \mathrm{y}$ \\ Luna Ma MANTOS ROLDÁN $^{4}$
}

\section{RESUMEN}

El presente trabajo pretende aportar información del grado de internacionalización de las cooperativas agrarias españolas, de los mercados y canales que están utilizando en la apertura de mercados exteriores. Se analiza además un caso real de empresa, la Sociedad Cooperativa Andaluza del Valle de los Pedroches (COVAP) que se está convirtiendo en un referente de empresa que ha sido capaz de llevar sus productos a más de 23 países entre los que se encuentran mercados tan exigentes como es Estados Unidos.

Palabras clave: cooperativa agroalimentaria, productos ganaderos, estrategia exportación, resultados exportadores.

Claves ECONLIT: M100, P130, Q170

\footnotetext{
${ }^{1}$ Debemos dejar constancia de nuestro agradecimiento por la ayuda recibida de la Agencia Andaluza de Promoción Exterior-EXTENDA al Director de Internacionalización de COVAP D. Abel Rodríguez y a los revisores de REVESCO por sus sugerencias.

${ }^{2}$ Facultad de Derecho y CC.Económicas y Empresariales- c/Puerta Nueva s/n 14071 Córdoba. Dirección de correo electrónico: fernando.fuentes@uco.es

${ }^{3}$ Profesora Contratada Doctora de Organización de Empresas en la Universidad de Córdoba. Dirección de correo electrónico: sandra.sanchez@uco.es

${ }^{4}$ Becaria del Área de Organización de Empresas en la Universidad de Córdoba. Dirección de correo electrónico: luna.santos@uco.es
}

REVESCO No 104 - Primer Cuatrimestre 2011 - ISSN: 1885-8031 - www.ucm.es/info/revesco

DOI: 10.5209/rev_REVE.2011.v104.2

Fecha de recepción: 19/05/2010

Fecha de aceptación: 03/11/2010 


\title{
FOOD AND AGRICULTURE COOPERATIVE AND EXPORTATION. THE PROCESS OF INTERNATIONALIZATION OF COVAP COOPERATIVE (POZOBLANCO-SPAIN)
}

\begin{abstract}
The current work is intended to widen the information related with the internationalization of the Spanish food and agriculture's cooperatives, as well as the markets and logistics in use to opening up to new markets. Moreover, this work analyses the case of "Sociedad Cooperativa Andaluza del Valle de los Pedroches (COVAP)", which is becoming a reference as they were able to sell their products within 23 countries, including the demanding US market.
\end{abstract}

Keywords: food and agriculture cooperative, farm products, export strategy, export results

\section{INTRODUCCIÓN: SECTOR AGROALIMENTARIO Y MOVIMIENTO COOPERATIVO.}

El sector agroalimentario se enfrenta a un entorno competitivo complejo y difícil, en el que el bajo crecimiento del consumo y la abundancia de oferta presionan constantemente los precios a la baja $\mathrm{y}$, aunque las empresas españolas presentan un buen nivel de calidad, seguridad alimentaria y competitividad también acusan importantes debilidades por su reducido tamaño y deficiente orientación al mercado (Boccherini: 2010).

Tradicionalmente la forma más habitual de combatir la atomización empresarial en nuestro sector primario ha sido la creación de cooperativas, organizaciones empresariales que aglutinan socios que voluntariamente y democráticamente acuerdan objetivos y que en este sector habitualmente se ocupan de almacenar, transformar, comercializar y, en definitiva, permitir que pequeños productores incrementen su poder de negociación y alcancen economías de escala y de alcance a las que individualmente no tendrían acceso. En España el movimiento cooperativo se inició en el siglo XIX pero fue en la segunda mitad del siglo XX 
cuando se produce el máximo desarrollo en paralelo a la modernización de la agricultura española. Según datos de la CCAE-CONFEDERACIÓN DE COOPERATIVAS AGRARIAS DE ESPAÑA(2010) en España operan un total de 3.900 cooperativas que facturaron 19.000 millones de euros en 2009 con un empleo directo cercano a los 100.000 trabajadores y un impacto indirecto muy superior (el número de socios total era ese año superior al millón de agricultores y ganaderos).

En este trabajo intentaremos aportar una revisión breve de las aportaciones más relevantes al estudio del comercio exterior de las cooperativas para a continuación mostrar un ejemplo de cómo una cooperativa agroganadera, de una comarca muy deprimida económicamente, ha sido capaz de formar una sólida empresa, gestionada de manera innovadora que en pocos años ha sido capaz de abrir numerosos mercados exteriores con lácteos, jamón ibérico y otros productos de comercialización difícil.

\section{MARCO TEÓRICO: INTERNACIONALIZACIÖN EMPRESARIAL Y SITUACIÓN DEL COOPERATIVISMO AGROALIMENTARIO}

\subsection{Teorías sobre la internacionalización}

El comercio internacional es un fenómeno que ha sido estudiado ya desde el mercantilismo y la escuela clásica (Adam Smith y David Ricardo). Los mercantilistas principalmente se ocuparon de estudiar las ventajas que obtienen los países en los intercambios comerciales pero no profundizaron en sus causas ni en el impacto en empresas concretas.

Posteriormente ya en el siglo XIX y el inicio del siglo XX se producen las aportaciones neoclásicas entre las que destacamos a Marshall, Barone, Lerner y Leontief. Los suecos Heckscher y Ohlin (1968) elaboraron un modelo predictivo (después mejorado por Samuelson) que analiza el efecto de la ventaja comparativa y competitiva que hará que los países tienden a exportar los bienes que son intensivos en los factores con que están abundantemente dotados. 
Tras la segunda guerra mundial, en paralelo con décadas de fuerte incremento del comercio mundial, se han producido importantes aportaciones teóricas. Ciñéndonos sólo a las relacionadas con la apertura al exterior de las empresas son reseñables:

- Teoría de la organización industrial (Hymer 1960) que afirma que las empresas se convierten en multinacionales porque determinadas compañías poseen ciertas ventajas competitivas (ej. mejores técnicas de producción, posibilidades de obtener economías de escala, diferenciación de productos...) que les inducen a la expansión internacional.

- Teoría de la internacionalización (Coase 1937).- Se basa en la teoría de los costes de transacción, la internacionalización se producirá si los beneficios derivados de la misma, como serían los costes de transacción, son superiores a los costes de aprendizaje y establecimiento que conlleva la expansión exterior.

- Teoría del ciclo de vida del producto (Vernon 1966) que considera que la ubicación de la empresa varía en función del grado de estandarización del producto ofertado. Cuando la diferenciación del producto confiere a la empresa una ventaja monopolística, esta decide exportarla primeramente a países desarrollados $\mathrm{y}$, cuando se pierde paulatinamente la posición oligopolística la compañía debe abandonar el producto o buscar una nueva ubicación donde los costes sean difícilmente alcanzables por sus competidores.

- Teoría de la diversificación de riesgos (Rugman 1981) sostiene que la motivación de la empresa que se internacionaliza es diversificar las inversiones con lo que los riesgos se diversifican y se protege de los riesgos derivados de coyunturas negativas específicas de cada país.

- Teoría ecléctica (Dunnig 1977), pretende integrar las teorías anteriores entendiendo que ninguna por separado es completa. Considera que una empresa se decidirá a invertir en el extranjero si concurren tres factores, propiedad de activos tangibles o intangibles, capaces de ser invertidos de forma rentable, que la localización de destino ofrezca ventajas frente a la permanencia en el mercado doméstico; que las empresas encuentren ventajoso explotar los activos ellas mismas en ese otro país. Esto es lo que se llama denomina paradigma OLI (Ownership, location, internationalization).

- Modelo de Uppsala (Johanson y Valhne 1977), surgido en la universidad sueca de Uppsala sostiene que las pymes inician su actividad en un país y paulatinamente la lógica de diversas decisiones incrementales pueden conducir a la internacionalización:

1- Ventas esporádicas en el exterior; 
2- Exportaciones a través de representantes independientes;

3- Establecimiento de sucursales independientes;

4- Establecimiento de unidades productivas en el exterior.

Este modelo predice que en un país concreto la empresa incrementará gradualmente los recursos comprometidos en el mismo en la medida que vaya adquiriendo conocimiento de ese mercado (Johanson y Valhne 1990).

Como afirma Grant (2006) la internacionalización es una de las fuerzas más importantes y generalizadas que ha cambiado el entorno competitivo de los negocios, pues ha permitido abrir los mercados nacionales a nuevos competidores, a la vez que ha creado nuevas oportunidades de negocio, tanto para pequeñas como grandes empresas. Claver y Quer (2000) aportan una visión integradora de la internacionalización en el seno de los procesos de Dirección Estratégica. Buscar nuevos mercados y tomar la decisión de internacionalización son estrategias de crecimiento y aprovechamiento de recursos y capacidades. La selección del modo de entrada en los mercados exteriores es una decisión clave en la estrategia de internacionalización de la empresa (Calderón y otros,2007). Los pasos más comunes en este proceso son: la exportación, el comercio y la inversión directa (Leonidou, 1995; Morello, 2001); como se ha anotado, la venta de productos en el exterior suele ser el paso inicial.

En el cuadro 1 se reflejan las causas de índole interna y externa que hacen que las empresas se abran al comercio exterior y a la inversión directa en el extranjero. Pla y León (2004) aportan otra clasificación de factores de apertura al exterior, denominados push y pull:

- Factores push.- los asociados a las dificultades de los mercados locales (saturación del mercado local, fuerte competencia doméstica...)

- Factores pull.- relacionados con la visión proactiva de los directivos. Aunque en los mercados domésticos queden oportunidades se estima que hay mercados emergentes dónde se pueden obtener ventajas. 
Cuadro 1. Causas que motivan la internacionalización empresarial.

\begin{tabular}{|l|l|}
\hline Internas & Externas \\
\hline Reducción de costes & Ciclo de vida de una industria \\
Tamaño mínimo eficiente & Demanda externa \\
Búsqueda de recursos & Pautas del consumidor \\
Disminución del riesgo global & Restricciones legales \\
Costes de transacción & Globalización de los mercados \\
Explotación de recursos y capacidades & \\
& \\
\hline
\end{tabular}

Fuente: Guerras y Navas (2007)

\subsection{Aportaciones $y$ problemática de las cooperativas agroalimentarias frente al comercio exterior}

A pesar de que el movimiento cooperativo en el sector primario constituye un objeto de estudio de gran impacto, en la economía española no son frecuentes los estudios publicados. Entre las aportaciones más recientes sobre la problemática del sector cabe destacar los siguientes trabajos:

- Vargas (2001) ha revisado las oportunidades de colaboración entre cooperativas transfronterizas analizando la colaboración entre empresas andaluzas y portuguesas.

- Vargas y García (2003) estudian el desempeño de cooperativas agrarias de Huelva y Jaén destacando que la gerencia de estas cooperativas guía sus actuaciones en primer lugar por ofrecer a los socios unos precios de liquidación de cosechas elevados por delante de reforzar la solidez financiera de la empresa.

- Sabaté (2003) por su parte analiza la eficiencia de cooperativas fruteras y el funcionamiento de una entidad (ACTEL S.C.L.) que ha sido capaz de integrar a 130 cooperativas de fruta, cereal y compras de suministros.

- El artículo de Ruiz Jiménez y otros (2006) aporta una revisión del estado de la investigación sobre cooperativas agrarias y sus estrategias destacando la imperiosa necesidad de crecimiento en un mercado tan competitivo como el actual y de la internacionalización. 
- En los últimos años parece imparable la tendencia a la creación de cooperativas de segundo grado lo que supone un incremento del poder de negociación en los mercados y alcanzar economías de escala inasequibles a las cooperativas más pequeñas. Martín (2006) analiza este fenómeno para varias cooperativas porcinas y Fernández y otros (2008) analizan los factores de éxito en el avance de la exportación en una muestra de cooperativas vinícolas. Resaltan estos autores que las exportaciones contribuyen a mejorar la utilización de la capacidad productiva de la empresa, a desarrollar capacidades de gestión superiores, a mejorar la calidad de procesos e innovar y a fortalecer resultados.

El proceso de concentración empresarial vigente hoy en día en el cooperativismo español supone que la integración horizontal permite también un incremento de la integración aguas abajo, es decir, que las cooperativas accedan a controlar los últimos eslabones de la cadena de valor de los productos agrarios incrementando el valor añadido con el que remunerar a los agricultores y ganaderos. Comienza a ser importante el volumen de producto con marca propia y el volumen de las exportaciones. Esta evolución lógica responde al citado modelo de Uppsala (Johanson y Vahlme, 1977), un enfoque teórico que explica la lógica de cómo las pymes van en paralelo a su crecimiento gradualmente incorporándose a los procesos de internacionalización.

La Confederación de Cooperativas Agrarias de España (2010) indica que en 2009 el $35 \%$ de las cooperativas españolas vende en otros países de la Unión Europea e incluso el $15 \%$ exporta a otros países no comunitarios. En el caso de las cooperativas de segundo grado de mayor tamaño (las que superan el millón de euros de facturación) la cifra de exportadoras aumenta hasta el $42,20 \%$, con un promedio de 12,47 millones de euros exportado por empresa.

Según las estadísticas publicadas, la implantación exterior de las cooperativas españolas es escasa, sólo el 7,41\% de las cooperativas de segundo grado se han establecido en el exterior y apenas la mitad en las cooperativas de primer grado. Precisamente Boccherini (2010) en relación a la agroindustria alimentaria española destaca que presenta un escaso interés por la internacionalización. La implantación en el exterior es ciertamente una tarea compleja, no exenta de riesgos y dificultades y muy consumidora de recursos directivos (tan escasos en las cooperativas). De hecho, la baja internacionalización se debe, en muchos casos, 
a los problemas de dimensión y formación empresarial. Por ello, nuestras cooperativas agrarias utilizan principalmente como canal de distribución intermediarios extranjeros (57\%) o españoles (entre el 45 y $49 \%$ según tipo de cooperativa). Sólo el 26\% venden directamente a cadenas de distribución extranjeras y un exiguo 3-5\% se distribuye en las delegaciones o empresas propias en el exterior.

Cuadro 2. Exportación en las cooperativas agrarias españolas.

\begin{tabular}{|l|c|c|}
\hline \multicolumn{1}{|c|}{ Cooperativas } & \multicolumn{1}{|c|}{$\begin{array}{c}1^{\circ} \text { grado } \\
>1 \mathrm{M} € \text { vtas. }\end{array}$} & \multicolumn{2}{|c|}{$2^{\circ}$ grado } \\
\hline Porcentaje de cooperativas exportadoras & 33,60 & 42,20 \\
\hline Exportación promedio de las cooperativas & 4,73 & 12,47 \\
exportadoras (M €) & & \\
\hline Porcentaje de implantación exterior (\%) & 3,67 & 7,41 \\
\hline Canales de exportación (\%) & & \\
\hline A través de otras cooperativas & 23,76 & 15,79 \\
A través de intermediarios españoles & 49,50 & 45,61 \\
A través de importadores o intermediarios extranj. & 57,42 & 56,14 \\
Directamente a cadenas de distribución extranjeras & 26,33 & 26,31 \\
A través de empresas o deleg. propias en el exterior & 5,94 & 3,51 \\
Otros canales de exportación & 7,92 & 17,54 \\
\hline
\end{tabular}

Fuente: $\operatorname{CCAE(2010)}$

Según se aprecia en el gráfico 1, por destinos, nuestras cooperativas fundamentalmente exportan a mercados de proximidad de la eurozona (Alemania, Francia, Portugal y Holanda) y al Reino Unido. Además las cooperativas de segundo grado han conseguido llegar a mercados más lejanos (Japón supera el $21 \%$ de volumen exportado y apenas el $11 \%$ a EEUU). Los grandes mercados de Rusia o China aún absorben muy modestas exportaciones de estas empresas. 
Gráfico 1. Destino de las exportaciones de las cooperativas españolas.

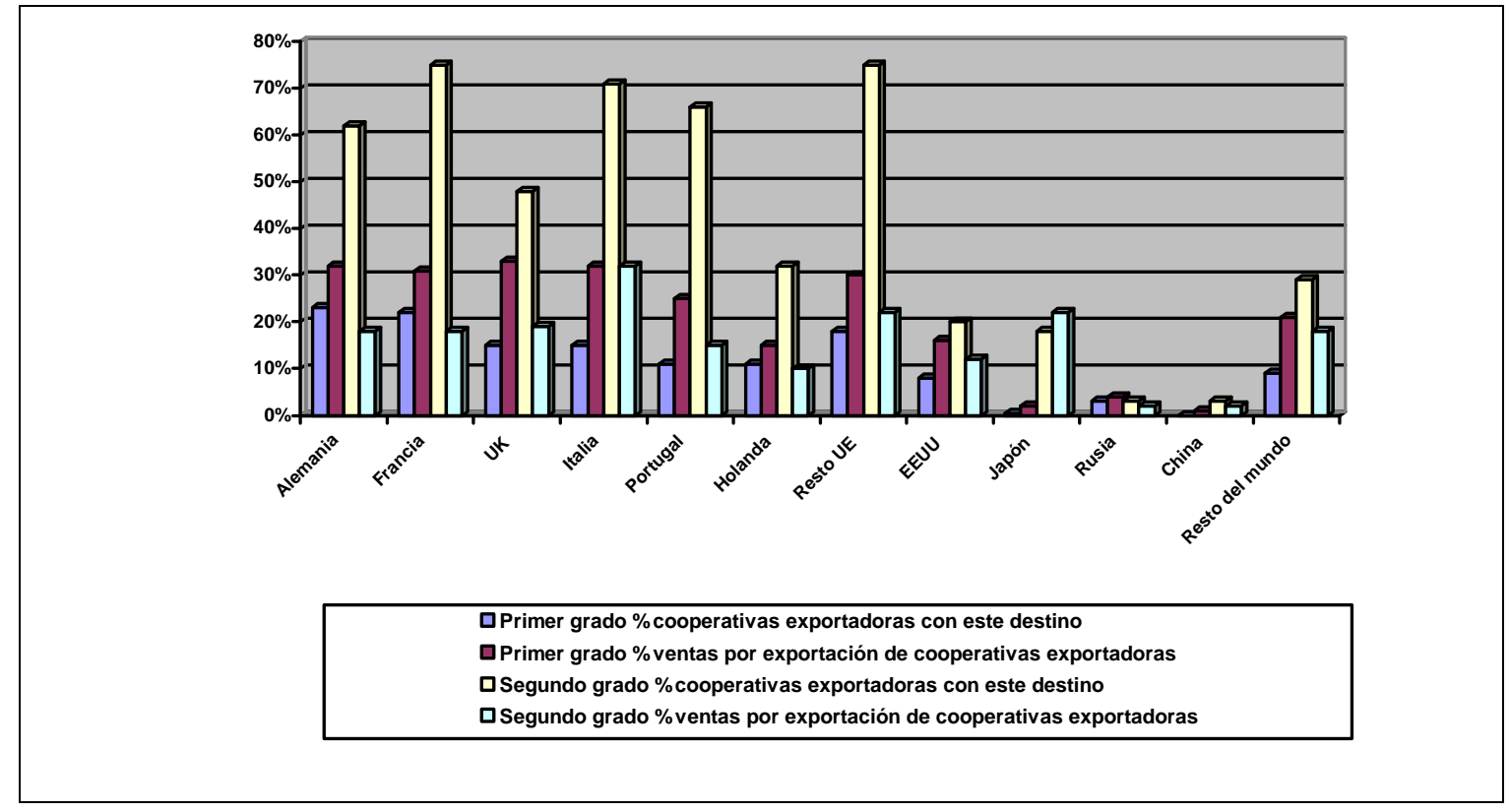

Fuente: $\operatorname{CAAE(2010)}$

Entre los escasos trabajos publicados en relación a las estrategias de exportación de las cooperativas se encuentra el reciente estudio de Fernández y otros (2008) que destacan que el avance en las ventas exteriores de las cooperativas vitivinícolas están relacionadas con la estrategia de liderazgo en costes y la diversificación de mercados entendida como la no concentración del esfuerzo en un mercado sino en atacar muchos países.

\section{LA INTERNACIONALIZACIÓN DE LA COOPERATIVA DEL VALLE DE LOS PEDROCHES (COVAP)}

\subsection{Historia de COVAP}

El origen de esta Cooperativa se remonta al año 1959 en que un grupo de 10 ganaderos de la localidad de Pozoblanco, en el Valle de Los Pedroches (Córdoba) deciden asociarse para la adquisición en mejores condiciones de pago, de una partida de maíz como alimento para su ganado. Esta asociación coyuntural se convertiría en el germen de lo que finalmente se consolidó en cooperativa. El proyecto inicial de la misma sería la construcción de una fábrica de piensos que se lleva a término en el año 1963, ampliándose de ese modo el ámbito de actuación de la Cooperativa y permitiendo su posterior desarrollo. Más adelante los socios comprendieron que era necesario afrontar el problema de la distribución de sus productos 
perecederos y que debido a la lejanía de Pozoblanco de los circuitos de distribución era conveniente que también la Cooperativa se ocupara de la transformación y la comercialización.

Así, los años siguientes son un claro exponente de la continua evolución en la gama de productos y servicios desarrollados por Covap, con la progresiva construcción y modernización de plantas y centros de tipificación. Son ejemplos significativos de este desarrollo la instalación de centros de recogida de leche en 1976, la creación del Matadero e Industria del cerdo ibérico en 1986 o la inauguración de la Sección de Crédito en 1996, entre otros múltiples proyectos.

En la actualidad, las explotaciones de los socios de Covap se ubican en tres áreas limítrofes del Norte de Córdoba (Valle de los Pedroches), Ciudad Real (Valle de la Alcudia) y Badajoz (Valle de la Serena). Se trata de una zona deprimida con grandes llanos de pastizal, mejores para la actividad ganadera en tanto los suelos son poco productivos para la agricultura.

La Cooperativa alcanza en el momento presente los 15.000 socios, lo que supone más del doble de la cifra de cooperativistas con que contaba en 1990 (6.067). La Asamblea General de Delegados elegidos en Juntas Preparatorias es el órgano que representa la voz de los socios en el seno de la Cooperativa. Es esta Asamblea la que nombra al Consejo Rector (incluido el presidente) que ejerce de órgano decisorio. Asimismo, cada una de las actividades tiene una comisión constituida por ganaderos de la misma y que representan a las distintas zonas donde opera la Cooperativa, teniendo como misión elevar distintas propuestas al Consejo Rector para su aprobación.

En 2006 se produjo un intento de transformación de la estructura societaria por las dificultades políticas, estratégicas y financieras para el desarrollo de los negocios que podía presentar una forma de Cooperativa. Se pensó entonces en un cambio en la organización para afrontar con éxito los nuevos retos de mercado mediante la transformación en Sociedad Anónima. Sin embargo, un grupo de socios, que se habían agrupado bajo la plataforma “Todos con Covap", cifrada en torno al 60 \% del total de cooperativistas, rechazó el proyecto al entender que suprimiría el "carácter fundacional de la cooperativa" y conllevaría una 
"quiebra importante" de la economía social en las comarcas en las que opera. Con ello se demuestra la vocación cooperativista de los socios.

En 2009, con motivo del 50 Aniversario de la fundación de la Cooperativa, su Presidente, Ricardo Delgado aboga por la idea de que Covap gane más dimensión empresarial para competir y sobrevivir, insistiéndose en la necesidad de que las cooperativas lleven a cabo alianzas "independientemente del lugar donde unas y otras estén ubicadas, incluso de índole internacional, porque es la única manera de seguir adelante dado la situación de crisis que actualmente estamos viviendo".

En estos 50 años, Covap ha pasado de ser una más de la multitud de cooperativas que surgieron en toda España a convertirse en el motor de desarrollo de la zona norte de la provincia de Córdoba. Puede afirmarse que Covap es hoy la cooperativa más importante de Andalucía, controlando gran parte del mercado andaluz de la leche y la carne y alcanzando una cifra de negocio que rondó los 300 millones de euros en 2009.

\subsection{Modelo de negocio de COVAP}

Según se aprecia en la figura 1, Covap ha basado su modelo de negocio en la forma de Sociedad Cooperativa con una preocupación constante por la figura del socio. Sus tres áreas funcionales presentan un alto grado de integración vertical. La sección de suministros se encarga del abastecimiento de piensos, materias primas y mezclas a los socios ganaderos de la organización. Asimismo, desde el área de servicios complementarios se ofrece un completo asesoramiento técnico, así como una sección de crédito, destinados en ambos casos al apoyo permanente al ganadero.

De hecho, Covap está desarrollando una labor de protección para sus socios en la actual crisis económica. Y es que entre los principios que basa su actuación se encuentran: el servicio a los socios, "trabajando para resolver sus problemas y hacer por ellos lo que individualmente no son capaces"; la mejora de sus rentas, abaratando los suministros y transformando y comercializando la producción; la voluntariedad, dado que los socios poseen libertad para asociarse a aquellas actividades que deseen; el pago por calidad, no habiendo diferencias por cantidades o distancias en lo que paga el socio por productos o servicios, pero existiendo un sistema de bonificaciones y penalizaciones en el pago al socio por los productos 
entregados, lo que se traduce en un estímulo para los cooperativistas y una garantía de calidad para el consumidor.

Por otro lado, las industrias alimentarias Covap, organizadas en función de la cabaña de ganado (vacuno de carne y de leche, caprino, ovino y cerdo ibérico) cuentan con una serie de instalaciones y centros en continuo proceso de crecimiento y sucesivas ampliaciones. El plan de inversiones 2000-2007 ha supuesto un volumen de 150 millones de euros y se ha traducido en el levantamiento de la planta láctea más moderna de Europa, un nuevo Centro de Tipificación de Corderos, un centro de cría de lechones, la ampliación del Centro de Tipificación de Terneros, un nuevo matadero, la ampliación de la industria del cerdo ibérico, una nueva planta de piensos y la puesta en marcha de una planta de platos preparados.

Este sistema altamente integrado verticalmente, permite a Covap realizar una apuesta decidida por la calidad, lo que ha trasladado de manera constante a sus métodos de trabajo y, de hecho, forma parte de su concepto de marca "Calidad desde el origen". El estricto control sanitario y alimenticio al que la cooperativa somete todos sus productos ha permitido que entre los logros de Covap destaque el hecho de haberse convertido en la primera empresa andaluza en obtener el certificado de calidad AENOR ISO 9002 para los productos lácteos. La fábrica de piensos y Centro de Tipificación de Terneros se encuentran acreditados por BVQI. Además, este centro, junto con el de Corderos, dispone de un sistema de certificación de productos cárnicos expedido por CERTICAR. Por su parte, la Planta de Piensos ha sido certificada por CESFAC. Asimismo, la empresa pertenece a la Asociación de Usuarios del Distintivo de Calidad Alimentos de Andalucía.

Entre sus objetivos estratégicos prioritarios Covap pretende llegar al cliente a través del mayor número de canales disponibles. De este modo, aparte de las redes de distribución habituales (cadenas de hipermercados y supermercados, mayoristas, hostelería y restauración, cadenas de charcuterías y carnicerías) la Cooperativa ha desarrollado una red de tiendas de calidad especializadas donde el consumidor puede adquirir directamente el producto Covap. 
Figura 1. Modelo de negocio COVAP

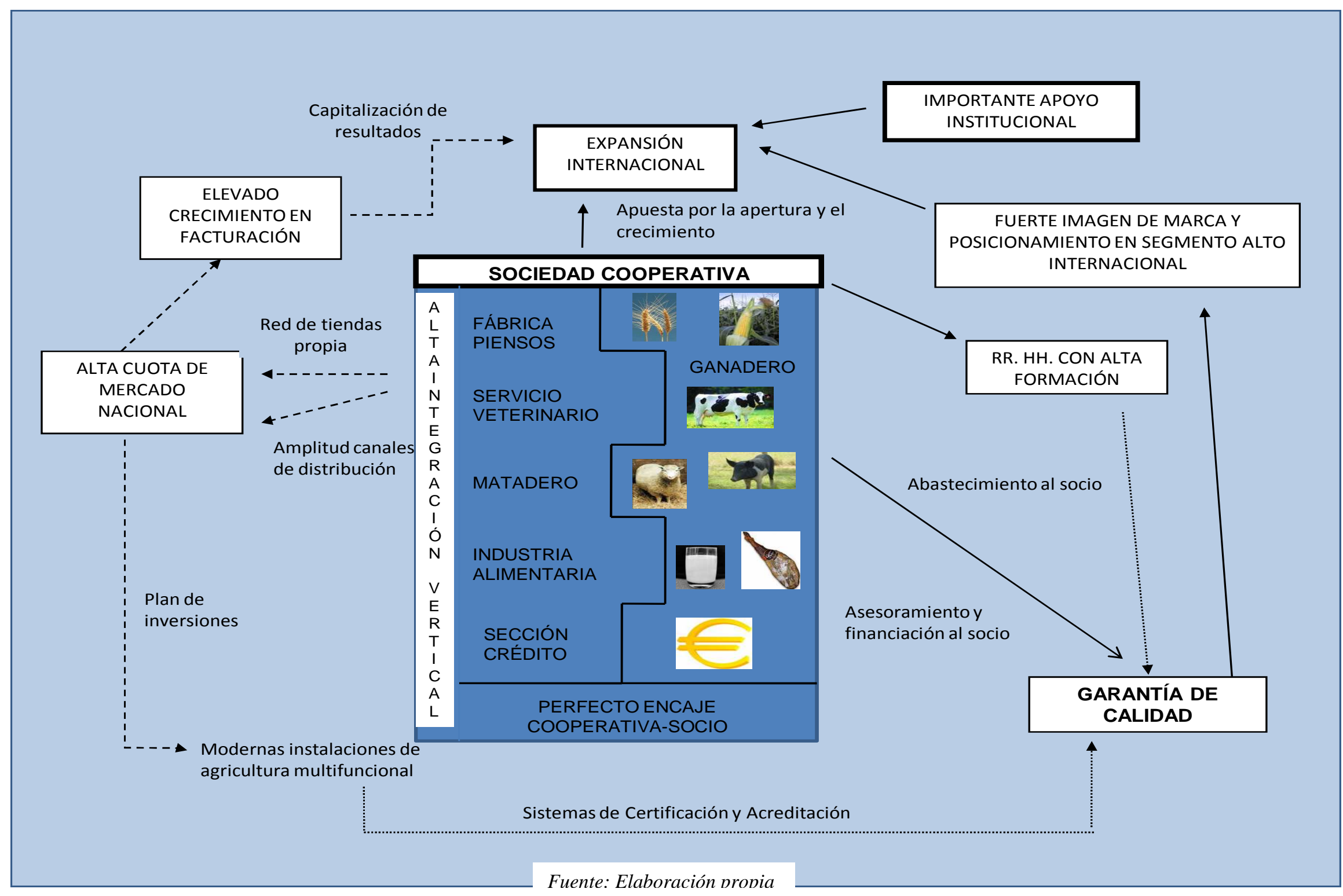

REVESCO No 104 - Primer Cuatrimestre 2011 - ISSN: 1885-8031 - www.ucm.es/info/revesco 
Con todo ello, el constante crecimiento en la facturación (gráfico 2) logrado por un sucesivo incremento de la cuota de mercado nacional, unido a la política financiera de reinversión de beneficios en un incremento permanente de activos, conforman las bases de la fortaleza de la cooperativa, según el director general, Miguel Angel Díaz Yubero. Para éste, las recientes cifras de ventas convierten a Covap en la cooperativa española de primer grado líder de facturación dentro del sector agroalimentario, y la quinta si se extiende el arco a las cooperativas de segundo grado.

Gráfico 2. Evolución ventas COVAP 1998-2009 (Millones de Euros)

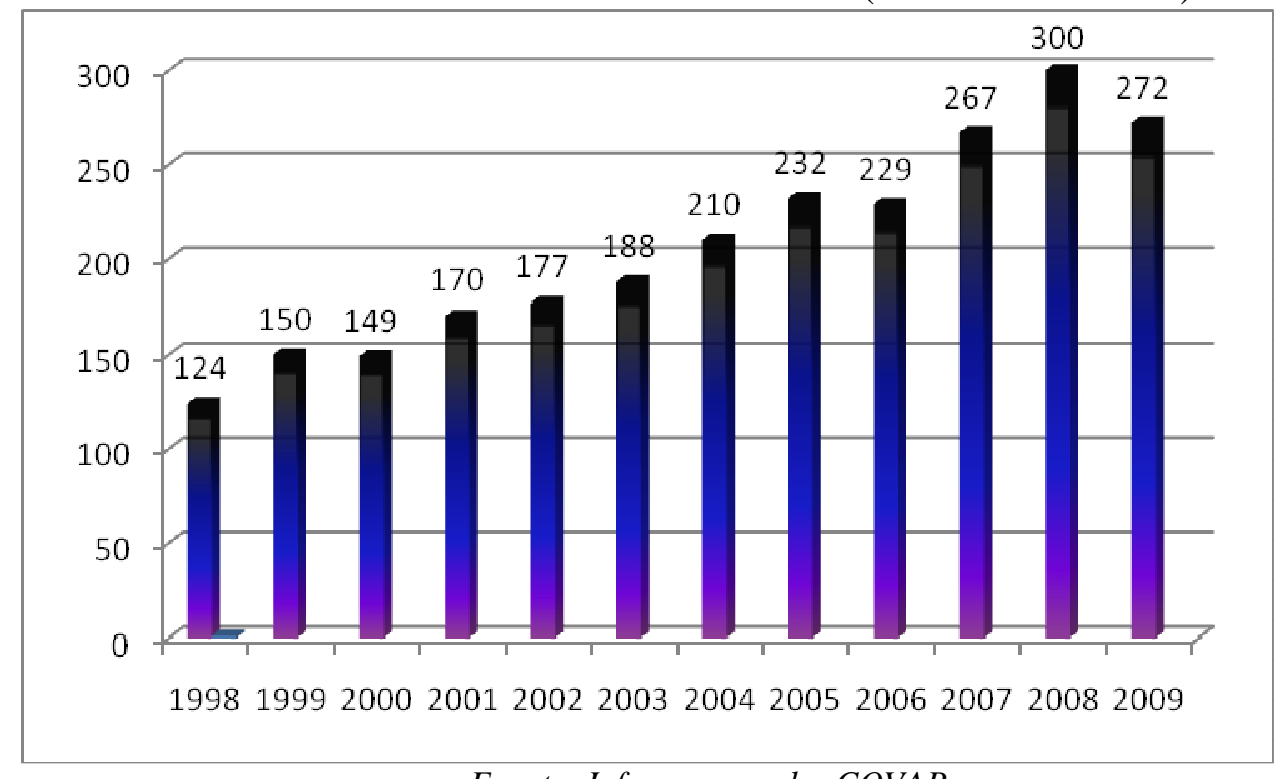

Fuente: Informes anuales COVAP

Estas cifras y el deseo de apostar por la apertura internacional llevaron a la Cooperativa a aventurarse en el mercado extranjero con la implantación en el año 2000 del Departamento Internacional. El proceso de internacionalización se analizará en más detalle en el siguiente epígrafe.

La estructura financiera de la Cooperativa apuesta por un equilibrio entre las partidas corrientes y no corrientes (gráfico 3), con un fondo de maniobra suficiente. Los recursos propios han pasado de una cifra de 12,2 a 92 millones de euros en los últimos veinte años. Unas cuentas permanentemente saneadas y un respaldo decidido por parte de distintas entidades y organismos públicos (en el caso del proceso de internacionalización cabe destacar el apoyo de Extenda o el ICEX) permiten a Covap gozar de una fortaleza económica con la que afrontar posibles escenarios de inseguridad financiera (tal y como sucede en el momento de crisis presente). 
Gráfico 3. Estructura de balance Covap.

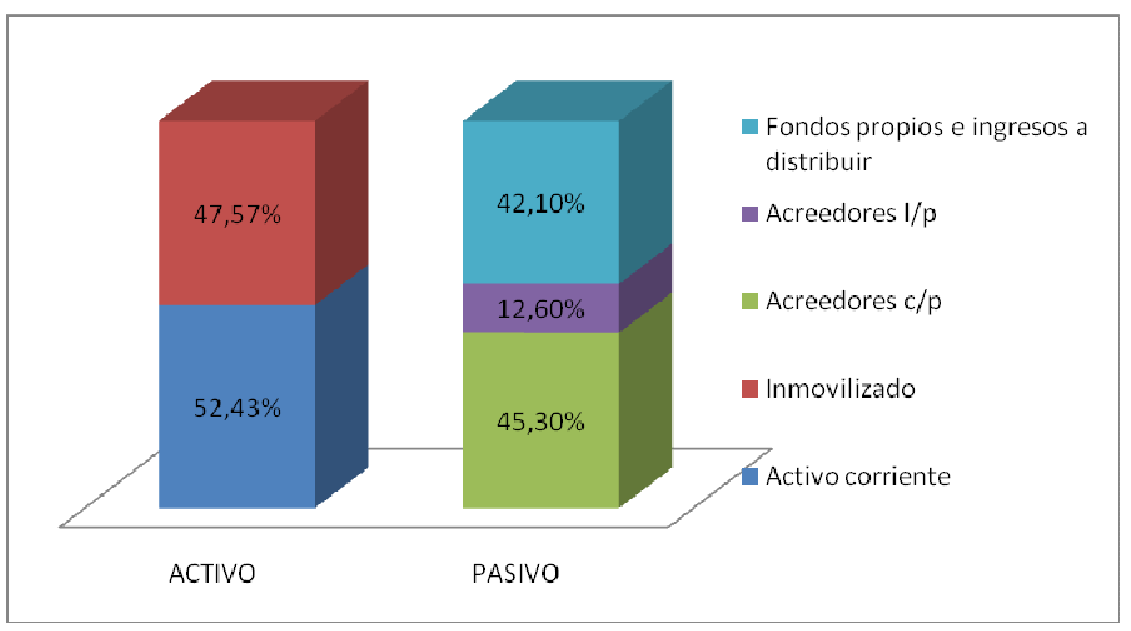

Fuente: Cuentas Anuales Covap y elaboración propia

Finalmente, pero no por ello menos importante, el Capital Humano con el que cuenta la Cooperativa se encuentra ampliamente formado y comprometido con la idea de calidad, gracias a un elevado porcentaje de estabilidad en el empleo. Este aspecto unido a todo lo anterior hacen que COVAP posea ya una andadura de 50 años plagados de logros, marque un referente en el sector agroalimentario español y, cada vez más, en el mercado internacional.

Cuadro 3. Elecciones clave en el modelo de negocio COVAP

\begin{tabular}{ll}
\hline DECISIONES & DESCRIPCIÓN \\
\hline Diseño & $\begin{array}{l}\text { Moderna agricultura multifuncional y respetuosa con el } \\
\text { medioambiente. } \\
\text { Protagonismo de los ganaderos }\end{array}$ \\
Amplio surtido de lácteos, cárnicos y platos preparados. \\
Suministro de piensos, mezclas y mercaderías al socio \\
que garantiza la calidad final del producto
\end{tabular}




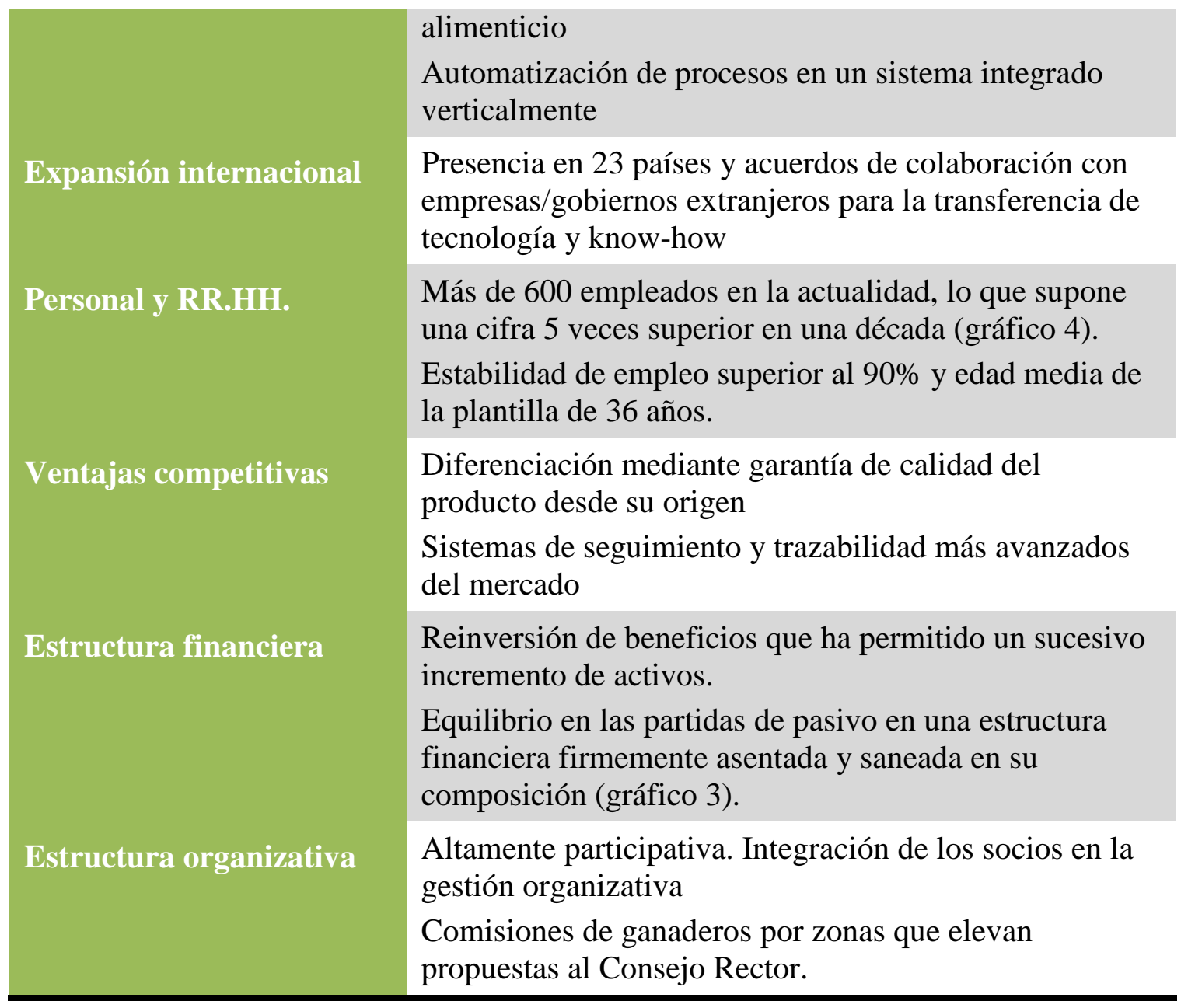

Fuente: Elaboración propia

Gráfico 4. Evolución de la cifra de empleados COVAP

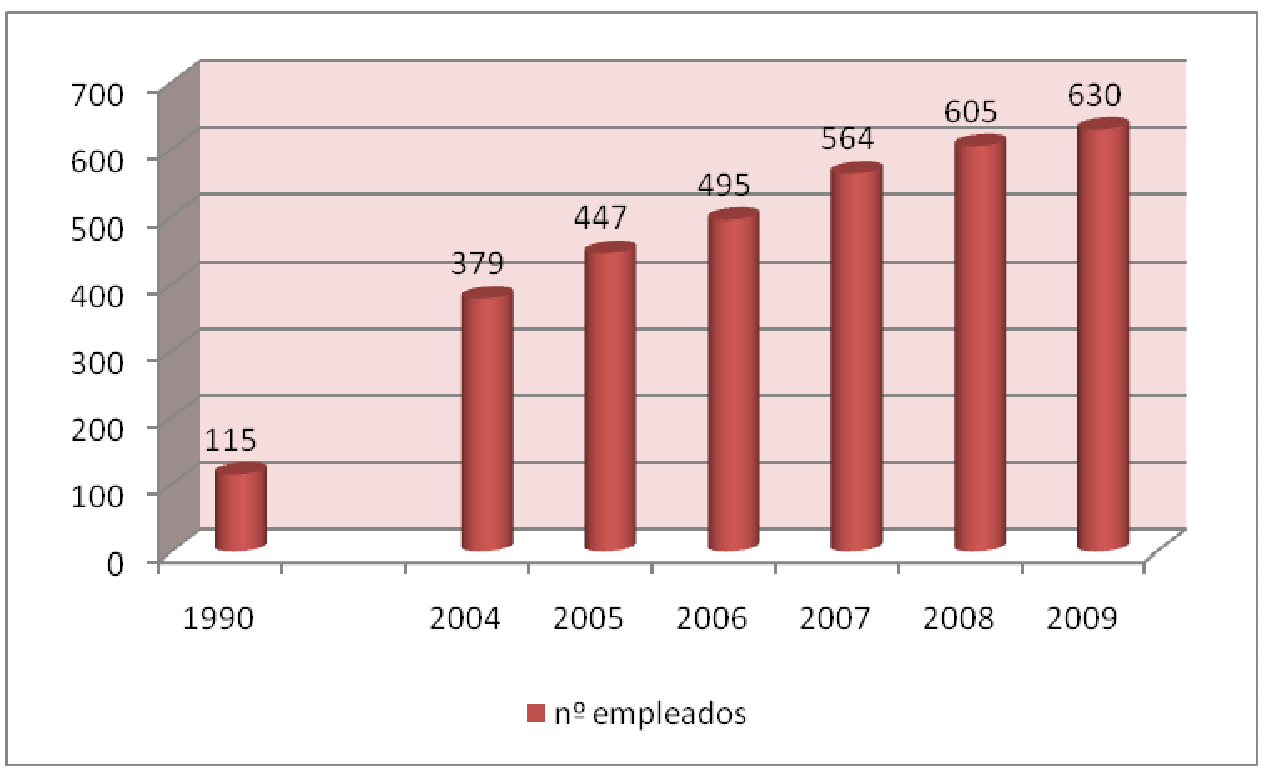

Fuente: Informes anuales COVAP 


\subsection{La internacionalización de COVAP}

Tal y como se mencionaba en el análisis del modelo de negocio, es en el año 2000 cuando se produce el inicio del proceso de internacionalización de Covap con la creación del Departamento Internacional. Son dos los principales objetivos que se marca desde el inicio de su andadura: por un lado, iniciar la trayectoria exportadora de la cooperativa, y en segundo lugar, alcanzar acuerdos de colaboración con empresas extranjeras para la transferencia de tecnología y know-how.

Según el Director del Departamento Internacional, Abel Rodríguez, puede afirmarse que en el período 2000-2009 los objetivos alcanzados han sido los siguientes:

- Apertura de nuevos mercados: presencia constante en 23 países en el 2008.

- Creación de la Marca COVAP, como referente de calidad agroalimentaria.

- Introducción en los canales apropiados de cada producto en los mercados exteriores.

- Acuerdos de colaboración con empresas / gobiernos extranjeros para la transferencia de tecnología y know-how.

La estructura organizativa de este departamento quedaría formada por dos áreas manager dependientes del Director. Por un lado, el área Europa del Este, Asia y Oceanía (desde abril de 2008) y por otro, el área América y África, aún pendiente de implantar. Existe asimismo un asistente para todas las áreas del que depende jerárquicamente un administrativo.

En cuanto a la evolución de las exportaciones, se puede afirmar que en menos de una década ha experimentado un auge importante gracias a la constante expansión de la cooperativa en distintos mercados internacionales. Tal y como se observa en el gráfico 5, el ritmo de crecimiento ha sido constante y especialmente significativo desde su inicio, salvo para el ejercicio 2007, donde se produjo un descenso del $23 \%$ en la facturación a causa de la apreciación del euro frente al dólar, el aumento en los precios de vacuno y ovino por la subida del precio del cereal y la disminución en la capacidad de suministros de nata a granel. 
Gráfico 5. Evolución exportaciones 2001-2009 (euros)

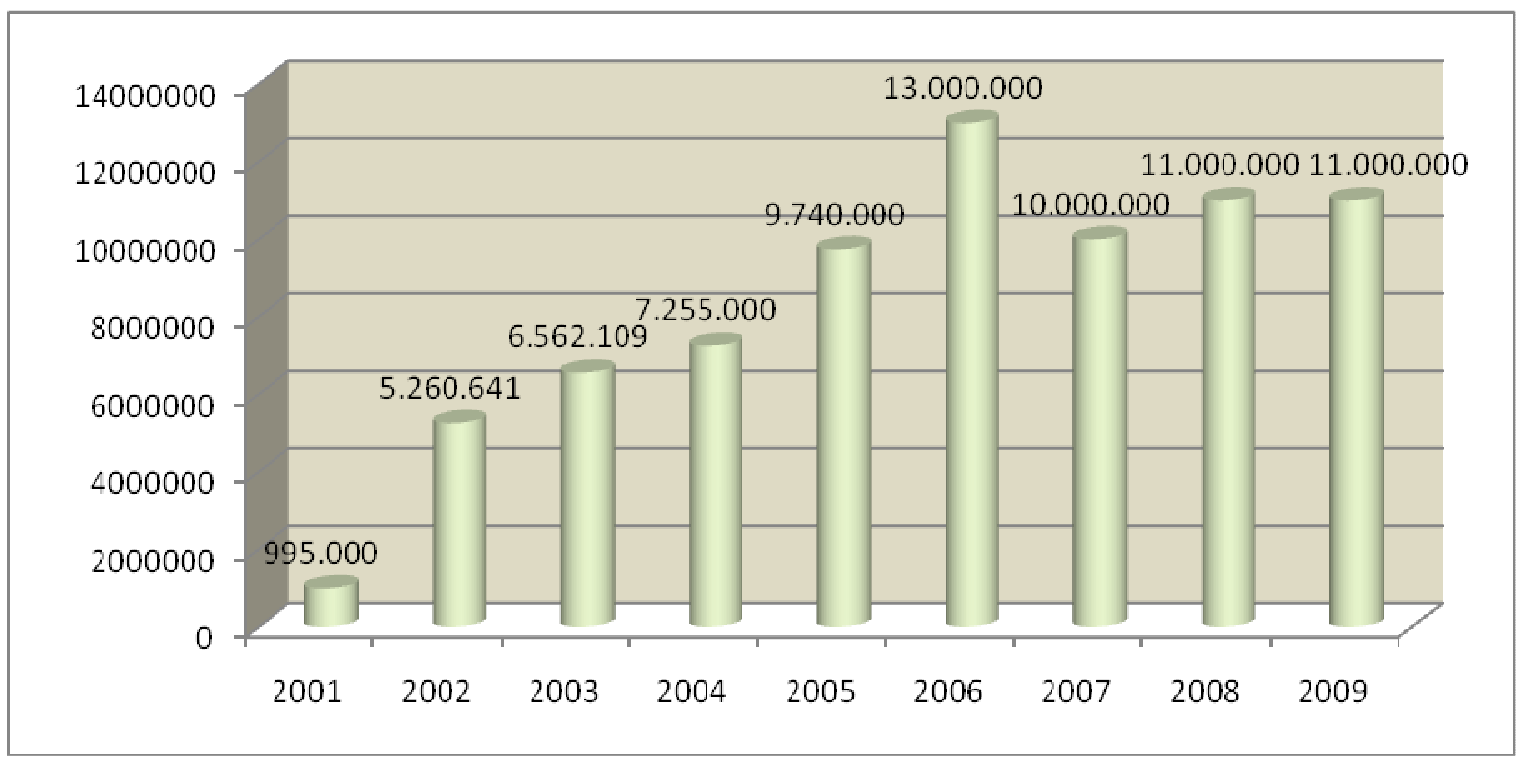

Fuente: Informes anuales COVAP

Los principales porcentajes de exportación se corresponden con los productos de ovino y vacuno, seguidos de los lácteos e ibéricos. En cuanto a los países de destino de las exportaciones en cada grupo, el cuadro 4 muestra cómo la Unión Europea es el destino preferente en todos los productos, especialmente en el caso de los ibéricos, que tienen presencia en 14 países del continente, así como en otros 6 países asiáticos y americanos. Los quesos forman otro grupo con amplia representación internacional. Igualmente, hay que destacar el nuevo grupo de platos preparados que se está abriendo camino en los mercados francés e italiano así como en Hong Kong.

Cuadro 4. Destinos de la exportación de productos COVAP

PRODUCTO EUROPA

Alemania, Austria, Bélgica,

Chequia, Francia, Grecia,

IBÉRICOS
Holanda, Irlanda, Italia,

Luxemburgo, Noruega, Suecia,

China, Hong

Chile, Martinica,

Kong, Japón México y EEUU

Portugal, Reino Unido 


\begin{tabular}{|c|c|c|c|}
\hline QUESOS & $\begin{array}{l}\text { Alemania, Chequia, Francia, } \\
\text { Holanda, Irlanda, Portugal, } \\
\text { Rusia }\end{array}$ & $\begin{array}{l}\text { China, Hong } \\
\text { Kong }\end{array}$ & $\begin{array}{l}\text { Chile, EE.UU., } \\
\text { Martinica, México }\end{array}$ \\
\hline OVINO Y & Alemania, Francia, Holanda, & & \\
\hline VACUNO & Italia, Portugal & & \\
\hline LÁCTEOS & $\begin{array}{l}\text { Francia, Grecia, Portugal, } \\
\text { Reino Unido }\end{array}$ & & \\
\hline PLATOS & Francia, Italia & Hong Kong & \\
\hline $\begin{array}{l}\text { LANA } \\
\text { MERINA }\end{array}$ & Reino Unido & & \\
\hline
\end{tabular}

Fuente: Informes anuales COVAP

En lo referente a volúmenes de facturación por países destacan en gran medida Italia y Portugal con exportaciones de 5,5 y 2,5 millones de euros respectivamente. Por otra parte, hay que subrayar la presencia de Japón, Hong Kong y Estados Unidos dentro de las diez primeras posiciones. En la actualidad, COVAP cuenta con 71 clientes extranjeros distribuidos en 23 países diferentes.

Gráfico 6. Principales países por porcentaje de exportación (10 primeras posiciones)

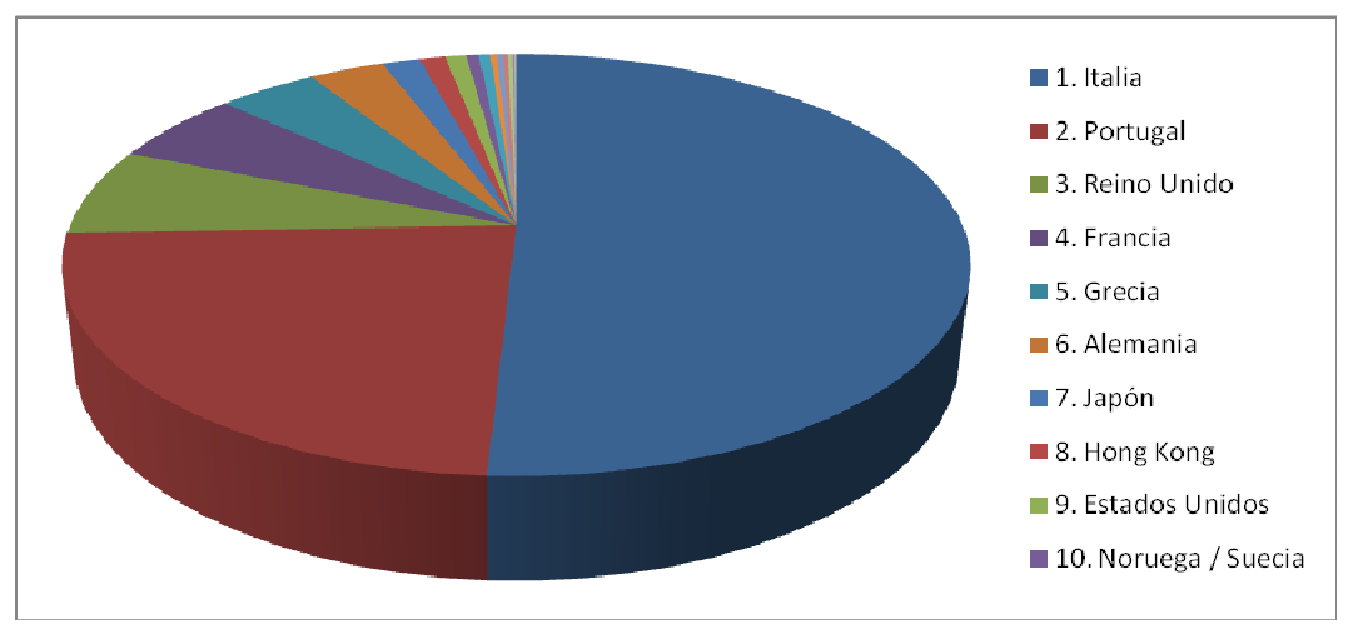

Fuente: Informes anuales COVAP 


\subsection{Retos de futuro}

Pese a la situación económica actual, Covap consiguió en 2008 un crecimiento del $21 \%$ para las ventas de leche, columna vertebral de la Cooperativa. Sin embargo, el sector de ibérico sufrió una caída de ventas del $10 \%$ arrastrado por el exceso de producción que la demanda no pudo absorber, generando además tensiones en los precios. En 2009, pese a la caída de precios incrementando las cantidades de producto vendidas se ha conseguido mantener la cifra de ventas.

Ante este escenario, Covap se plantea una serie de actuaciones a corto y medio plazo. Por un lado, se pretende plantear la modificación de los estatutos sociales de la entidad, que afectarán a cuestiones como los socios inactivos, la composición del consejo rector, las aportaciones a capital Social, o la distribución de los excedentes. Paralelamente, se ha anunciado la elaboración antes de un plan estratégico para el periodo 2010-13 cuyas líneas están por definir.

Por otro lado, Covap se ha incorporado recientemente como socio a la cooperativa de segundo grado Cordesur, constituida por Corsevilla y Dehesas Cordobesas (Corpedroches), como una plataforma donde se concentre la oferta con el ánimo de regular el mercado del ovino andaluz. También se está estudiando el planteamiento de alianzas con grandes cooperativas de alimentación andaluzas como Hojiblanca o Agrosevilla, pretenden incidir en la línea de abaratar costes y adquirir dimensión, que, en particular permitirán reforzar la presencia en mercados exteriores.

La apuesta de futuro de Covap se mantiene en la protección al socio mediante una estrategia permanente de crecimiento y, en palabras de su presidente, Ricardo Delgado, "una forma de insistir en este aspecto es potenciar las ventas en el mercado internacional", de ahí la radical importancia que cobra el proceso de internacionalización de la Cooperativa y la necesidad de continuar la línea de apertura a nuevos mercados.

En 2010 COVAP ha conseguido las autorizaciones que permitirán la exportación de sus productos de cerdo ibérico a Estados Unidos, un permiso que sólo tenía anteriormente otro matadero español. Los productos se irán introduciendo de forma 
escalonada ya que sólo se podrán exportar los ejemplares que hayan sido sacrificados después de obtener la licencia, a primeros de abril. Mientras que los embutidos podrán llegar a finales de este año, las paletas de cerdo ibérico no desembarcarán en Estados Unidos hasta 2011, tras un proceso de curación de 18 meses, y los jamones llegarán en 2012, con una curación de 24 meses. Se estima que el jamón ibérico curado podrá alcanzar los 350 dólares por kilo.

Las perspectivas de facturación de COVAP en Estados Unidos prevén alcanzar los 400.000 euros el primer año, un millón de euros en 2011 y 1,5 millones en el siguiente, de forma que en 2013 se puedan alcanzar los 2 millones de euros de ventas anuales con un elevado margen, sin tener en cuenta la venta de quesos, que la cooperativa cordobesa inició en el mercado estadounidense hace ya cinco años.

Para alcanzar estas cifras de negocio, la estrategia de ventas tiene previsto iniciar la comercialización por la Costa Este de Estados Unidos, que incluye zonas como Nueva York, Washington DC, Boston o Chicago, además de estados del Noroeste y los de Virginia, Maryland, California y Florida.

En estos mercados, la empresa desarrollará una labor de promoción e información de las bondades del ibérico, su corte, su presentación y su degustación y también contará con el apoyo del ICEX y la Agencia Andaluza de Promoción ExteriorEXTENDA.

Los ibéricos de COVAP se distribuirán a través de "Forever Cheese", que importa y distribuye productos gourmet en 45 de los 50 estados norteamericanos y ya comercializa el queso de la cooperativa en Estados Unidos; "Whole Foods", la cadena líder de supermercados y la más grande del mundo; "Agrosevilla Inc.", una cooperativa sevillana que trabaja con cadenas de pizzerías, y a través de "Tienda.com", que comercializará productos de cerdo ibérico loncheados a través de internet.

Igualmente también se han iniciado las exportaciones a Australia, en este caso de jamones ibéricos deshuesados a través de un importador local. 
También en 2010 y para la comercialización del cordero, la cooperativa Covap se ha unido a otras dos cooperativas (Dehesas Cordobesas y San Miguel) para crear una asociación promotora de la marca de calidad Cordero de los Pedroches, una iniciativa que tiene como objetivo la promoción del consumo de este tipo de carne con el marchamo de calidad garantizado en cuanto a origen, sistema productivo y trazabilidad.

Finalmente es de reseñar también la contribución como socio de referencia de la cooperativa COVAP junto otras empresas e instituciones públicas y la Universidad de Córdoba $^{5}$ a la recién creada Fundación CICAP (Centro de Investigación y Calidad Agroalimentaria de Los Pedroches-Córdoba) un centro especializado en I+D+i agroalimentario con el que se quieren desarrollar nuevos productos, procesos de elaboración, envases, etc.

\section{CONCLUSIONES}

Las empresas cooperativas agroalimentarias españolas no son ajenas a los problemas de los mercados de consumo(saturación del mercado local, fuerte competencia doméstica) y parece evidente que necesitan incrementar su dimensión frente a la fuerte presión de los países emergentes en el sector agro-ganadero (Polonia, República Checa, Rumanía, Bulgaria o Turquía), generar un mayor valor añadido y eliminar intermediarios entre productores y consumidores.

En una economía global como la actual y con una coyuntura interna de debilidad de la demanda interna, la apertura de mercados exteriores resulta imprescindible para el tejido empresarial e imprescindible para las cooperativas que comercializan productos de gran calidad.

Tradicionalmente, la gerencia de numerosas cooperativas agrarias ha tenido carencias formativas importantes en sus cuadros directivos y se ha enfrentado a la presión de los socios por retribuir el producto recibido a precios altos que dificultaban

\footnotetext{
${ }^{5}$ En este sentido tal universidad lidera a su vez el proyecto de Campus de Excelencia en Investigación Agroalimentaria $\operatorname{cei}_{\mathrm{A}} 3$ junto a las universidades de Huelva, Cádiz, Jaén y Almeria que pretende ser un referente en investigación y transferencia entre universidades y tejido productivo.
} 
las inversiones a largo plazo entre las que podríamos incluir la apertura de mercados exteriores.

En los últimos años se aprecia en el sector cierta proactividad con movimientos horizontales y verticales de fusiones de cooperativas de primer grado y de integraciones en cooperativas de segundo grado y de alianzas entre grupos de cooperativas de variados productos (podemos citar los ejemplos de Hojiblanca, Agrosevilla, Oviporc...) que pretenden incidir en la línea de abaratar costes y adquirir la dimensión suficiente para acceder a mercados exteriores.

Se ha analizado aquí, un caso quizá excepcional, que puede ser ejemplo de que es viable que las empresas cooperativas agroalimentarias se abran al exterior. En concreto se trata de la empresa Covap un buen referente de éxito en la integración estratégica y compromiso entre protección al socio y en paralelo de un crecimiento sostenido basado en instalaciones modernas, calidad en todos los procesos y explotación de marcas propias. Con ello se integran las expectativas de todos los grupos de interés: socios-propietarios-proveedores, clientes, trabajadores, directivos y sociedad en general. Esta cooperativa ha conseguido en diez años abrir mercados en 23 países entre los que se encuentran los más exigentes del mundo (como es por ejemplo Estados Unidos).

\section{BIBLIOGRAFIA}

ARCAS, N., MUNNUERA, J.L. y HERNÁNDEZ, M.J.(2002), "Beneficios de las cooperativas agrarias de segundo grado: contribución a los objetivos de sus socios", REVESCO, $\mathrm{N}^{\mathrm{o}} 76, \mathrm{PP} 7-26$.

BAHAMONDE, E.(2009), "Cooperativismo agroalimentario", Mediterráneo económico, $\mathrm{n}^{\mathrm{o}} 15$ pp.229-246.

BOCCHERINI, José Antonio (2010), "Nuevos retos competitivos para la cadena agroalimentaria española" pp. 17-27, en MERCASA (2010), Alimentación en España 2009, Ed. Ministerio de Medio Ambiente, Rural y Marino, Madrid.

CALDERÓN, H., CERVERA, A., TURILLEJAS, B. y FAYOS, T. (2007), “Selección del modo de entrada en un mercado internacional: valoración de las capacidades 
empresariales, la estrategia empresarial y la percepción de los problemas de la internacionalización", Tribuna de Economía ICE, 839: 143-162.

CALDERÓN, H. y FAYOS, T. (2004), "Factores empresariales que influyen en las políticas de promoción de las exportaciones: aplicación a la comunidad valenciana", Dirección y Organización, 30: 122-133.

CCAE-CONFEDERACION DE COOPERATIVAS AGRARIAS DE ESPAÑA (2010), “Observatorio socioeconómico del cooperativismo agroalimentario español Estadísticas sobre las cooperativas españolas, según datos relativos al ejercicio 2009", CCAE, Madrid.

CLAVER CORTES, E. y QUER RAMON, D. (2000): Estrategias de internacionalización de la empresa, Ed. Club Universitario.

COASE, R. H. (1937): “The nature of the firm”. Económica, 4, págs. 386-405.

DUNNING, J.H. (1977), 'Trade location of economic activity and the MNE: A search for an eclectic approach', en Ohlin, B., Hesselborn, P.D. and Wijkman, P.M. (ed.), The International Allocation of Economic Activity, London: Macmillan, págs. 395418.

DURAN, Juan José, 2001, Estrategia y economía de la empresa multinacional, Ed. Pirámide, Madrid, $621 \mathrm{pp}$.

FERNÁNDEZ MORENO, MaValle, PEÑA GARCÍA-PARDO, Isidro y HERNÁNDEZ PERLINES, Felipe (2008), "Factores determinantes del éxito exportador. El papel de la estrategia exportadora en las cooperativas agrarias", CIRIEC, $\mathrm{N}^{\circ} 63$, pp.39-64.

GIRALDEZ PIDAL, Elena, 2002, La internacionalización de las empresas españolas en América Latina, CES, Madrid, 239 pp.

GRANT, Robert, 2006, Dirección Estratégica, Ed. Cívitas, Madrid, 669 pp.

GUERRAS, L.A. y NAVAS LÓPEZ, J.E. (2007): La dirección estratégica de la empresa. Ed. Thompson-Civitas. Madrid.

GUISADO TATO, Manuel, 2002, Internacionalización de la empresa. Estrategias de entrada en Mercados Extranjeros, Pirámide, Madrid, 411 pp.

HECKSCHER, E. F. (1968): "The effect of foreign trade on the distribution of income", Readings in International Economics, editado por R. E. CAVES y H. G. Johnson. Homewood.

HYMER, S. H. (1960): The international operations of national firms: a study of direct foreign investment. The MIT Press, 1976. Cambridge, Mass. 
JOHANSON, J. y VAHLNE, J.E. (1977), “The Internationalization process of firms. A model of knowledge development and increasing foreign market commitens" Journal of internationational Business Studies n ${ }^{\circ} 8$, pp.22-32.

JOHANSON, J. y VAHLNE, J.E. (1990), “The Mechanism of internationalization" International Marketing Review, vol. 7-4, pp.11-24.

JOYNT, P.; WELCH, L. (1985): “A strategy for Small Business Internationalisation”. International Marketing Review, 2, nº 3, págs. 64-73.

LEONIDOU, L. C. (1995), "Export Simulation: A Non-exporter's Perspective", European Journal of Marketing, 29 (8): 17-36.

MARTÍN, Carmen (2006) "Cuatro cooperativas se unen para el fomento de la exportación de porcino", Mundo ganadero, n 186, pp. 28-31.

MARTIN ROJO, I. y GASPAR GONZALEZ, Ana Isabel, 2007, Crecimiento e internacionalización de empresas, Ed. Síntesis, Madrid, 215 pp.

MORELLO, G. (2001), “El proceso de internacionalización”, Economía y Desarrollo, 2 (129): 178-192.

OHLIN, B. 1933. Interregional and International Trade. Cambridge, Mass.: Harvard

University Press, 1966.

PLA BARBER, José y LEON DARDER, Fidel, 2004, Dirección de empresas internacionales, Ed. Pearson-Alhambra, Madrid, 384 pp.

RUGMAN, A.M. (1981). Inside the multinationals: the economics of internal markets. Columbia University Press, New York.

RUIZ JIMENEZ, MªCarmen, HERÁNDEZ ORTIZ, Ma Jesús y GARCÍA MARTÍ, Elia (2006), "Estado actual de la investigación sobre sociedades cooperativas en España”, CIRIEC, nº 56, pp.65-86.

SABATÉ PRATS, P.(2002), “Análisis comparativo de la eficiencia de las cooperativas fruteras de la provincia de Lleida", CIRIEC, Nº41, PP 163-182.

SMITH, A. (1776): La riqueza de las naciones. Madrid, Alianza Editorial.

VARGAS, A.(2001), "El cooperativismo agrario andaluz y la cooperación empresarial transfronteriza", REVESCO, $\mathrm{N}^{\circ} 75$, PP. 150-168.

VARGAS, A. y GARCÍA MARTÍ, E.(2003), "La medición del desempeño en las sociedades cooperativas agrarias. Perspectivas de los directores gerentes de Huelva y Jaén ”, CIRIEC, № 46 PP.85-116.

VERNON, R. (1966), "International investment and international trade in the product Cycle”. Quarterly Journal of Economics, 80, págs. 190-207. 